\title{
On Poisson-Weighted Lindley Distribution and Its Applications
}

\author{
R. Shanker, K. K. Shukla ${ }^{*}$ \\ Department of Statistics, College of Science, Eritrea Institute of Technology, Asmara, Eritrea
}

Received 26 May 2018, accepted in final revised form 18 July 2018

\begin{abstract}
In this paper the nature and behavior of its coefficient of variation, skewness, kurtosis and index of dispersion of Poisson- weighted Lindley distribution (P-WLD), a Poisson mixture of weighted Lindley distribution, have been proposed and the nature and behavior have been explained graphically. Maximum likelihood estimation has been discussed to estimate its parameters. Applications of the proposed distribution have been discussed and its goodness of fit has been compared with Poisson distribution (PD), Poisson-Lindley distribution (PLD), negative binomial distribution (NBD) and generalized Poisson-Lindley distribution (GPLD).
\end{abstract}

Keywords: Poisson lindley distribution; Weighted lindley distribution, Compounding; Skewness; Kurtosis; Maximum likelihood estimation.

(C) 2019 JSR Publications. ISSN: 2070-0237 (Print); 2070-0245 (Online). All rights reserved. doi: http://dx.doi.org/10.3329/jsr.v11i1.35745

J. Sci. Res. 11 (1), 1-13 (2019)

\section{Introduction}

M. Shankaran [1] proposed the Poisson-Lindley distribution (PLD) to model count data defined by its probability mass function (pmf)

$$
P_{1}(x ; \theta)=\frac{\theta^{2}(x+\theta+2)}{(\theta+1)^{x+3}} ; x=0,1,2, \ldots, \theta>0
$$

Shanker and Hagos [2] proposed a simple method of finding moments of PLD and discussed the applications of PLD to model count data from biological sciences. Shanker and Shukla [3] proposed a generalized size-biased Lindley distribution which includes size-biased Poisson-Lindley distribution introduced by Ghitany et al. [4] as special case and discussed its statistical properties, estimation of parameters and applications for modeling the distribution of freely-forming small group. The distribution arises from the Poisson distribution when its parameter $\lambda$ follows distribution [5] defined by its probability density function (pdf).

\footnotetext{
*Corresponding author: kkshukla22@gmail.com
} 


$$
f_{1}(\lambda ; \theta)=\frac{\theta^{2}}{\theta+1}(1+\lambda) e^{-\theta \lambda} ; \lambda>0, \theta>0 ; \quad x>0, \theta>0
$$

It can be easily verified that the pdf (1.2) is a two-component mixture of exponential ( $\theta)$ and gamma $(2, \theta)$ distributions. Ghitany et al. [6] discussed statistical properties including moments based coefficients, hazard rate function, mean residual life function, mean deviations, stochastic ordering, Renyi entropy measure, order statistics, Bonferroni and Lorenz curves, stress-strength reliability, along with estimation of parameter and application to model waiting time data in a bank. Shanker et al. [7] have detailed study on modeling of various lifetime data from engineering and biomedical sciences using exponential and Lindley distribution and observed that there are many lifetime data where exponential distribution gives much better fit than Lindley distribution.

The first four moments about origin and the variance of PLD (1.1) are given by

$$
\begin{aligned}
& \mu_{1}^{\prime}=\frac{\theta+2}{\theta(\theta+1)} \\
& \mu_{2}^{\prime}=\frac{\theta^{2}+4 \theta+6}{\theta^{2}(\theta+1)} \quad \mu_{3}^{\prime}=\frac{\theta^{3}+6 \theta^{2}+24 \theta+24}{\theta^{3}(\theta+1)} \\
& \mu_{4}^{\prime}=\frac{\theta^{4}+16 \theta^{3}+78 \theta^{2}+168 \theta+120}{\theta^{4}(\theta+1)}
\end{aligned}
$$

introduced a two-parameter weighted Lindley distribution (WLD) [8] having parameters $\theta$ and $\alpha$ and defined by its pdf

$f_{2}(x ; \theta, \alpha)=\frac{\theta^{\alpha+1}}{(\theta+\alpha)} \frac{x^{\alpha-1}}{\Gamma(\alpha)}(1+x) e^{-\theta x} ; x>0, \theta>0, \alpha>0$

where

$\Gamma(\alpha)=\int_{0}^{\infty} e^{-y} y^{\alpha-1} d y ; \alpha>0$

is the complete gamma function. Its structural properties including moments, hazard rate function, mean residual life function, estimation of parameters and applications for modeling survival time data has been discussed by Ghitany et al. [8]. The corresponding cumulative distribution function (cdf) of WLD (1.3) is given by

$$
F(x ; \theta, \alpha)=1-\frac{(\theta+\alpha) \Gamma(\alpha, \theta x)+(\theta x)^{\alpha} e^{-\theta x}}{(\theta+\alpha) \Gamma(\alpha)} ; x>0, \theta>0, \alpha>0
$$

where

$\Gamma(\alpha, z)=\int_{z}^{\infty} e^{-y} y^{\alpha-1} d y ; \alpha>0, z \geq 0$

is the upper incomplete gamma function. It can be easily shown that at $\alpha=1$, WLD (1.3) reduces [5] to distribution (1.2). Shanker et al. [9] discussed various moments based 
properties including coefficient of variation, coefficient of skewness, coefficient of kurtosis and index of dispersion of WLD and its applications to model lifetime data from biomedical sciences and engineering. Shanker et al. [10] have proposed a three-parameter weighted Lindley distribution (TPWLD) which includes one parameter exponential and Lindley distributions, two parameter gamma and weighted Lindley distributions as particular cases and discussed its various structural properties, estimation of parameters and applications for modeling lifetime data from engineering and biomedical sciences.

The main purpose of this paper is to discuss the nature and behavior of the coefficient of variation, skewness, kurtosis and index of dispersion of Poisson-Weighted Lindley distribution (P-WLD), a Poisson mixture of weighted Lindley distribution. Note that ElMonsef et al. [11] have discussed some properties of P-WLD including moments based measures but they have unnecessarily introduced hyper geometric function in the expressions of raw moments which is illogical. In fact, the raw moments and central moments are straightforward for P-WLD. Further, Monsef et al. [11] has claimed that it gives better fit as compared with other distributions, which is not correct even in their paper. Maximum likelihood estimation has been discussed to estimate its parameters. Applications of the proposed distribution have been discussed and its goodness of fit has been compared with Poisson distribution (PD), Poisson-Lindley distribution (PLD), negative binomial distribution (NBD) and generalized Poisson-Lindley distribution (GPLD).

\section{Poisson-Weighted Lindley Distribution}

Assuming that the parameter $\lambda$ of the Poisson distribution follows the WLD (1.3), the Poisson mixture of WLD can be obtained as

$$
\begin{aligned}
& P_{2}(x ; \theta, \alpha)=\int_{0}^{\infty} \frac{e^{-\lambda} \lambda^{x}}{x !} \frac{\theta^{\alpha+1}}{(\theta+\alpha)} \frac{\lambda^{\alpha-1}}{\Gamma(\alpha)}(1+\lambda) e^{-\theta \lambda} d \lambda \\
& =\frac{\theta^{\alpha+1}}{(\theta+\alpha) \Gamma(\alpha) x !\left[\int_{0}^{\infty} e^{-(\theta+1) \lambda} \lambda^{x+\alpha-1} d \lambda+\int_{0}^{\infty} e^{-(\theta+1) \lambda} \lambda^{x+\alpha+1-1} d \lambda\right]} \\
& =\frac{\theta^{\alpha+1}}{(\theta+\alpha) \Gamma(\alpha) x !}\left[\frac{\Gamma(x+\alpha)}{(\theta+1)^{x+\alpha}}+\frac{\Gamma(x+\alpha+1)}{(\theta+1)^{x+\alpha+1}}\right] \\
& \quad=\frac{\Gamma(x+\alpha)}{\Gamma(x+1) \Gamma(\alpha)} \frac{\theta^{\alpha+1}}{(\theta+\alpha)} \frac{x+\theta+\alpha+1}{(\theta+1)^{x+\alpha+1}} ; x=0,1,2, \ldots, \theta>0, \alpha>0
\end{aligned}
$$

We would call this pmf the Poisson-Weighted Lindley distribution (P-WLD). It can be easily verified that PLD (1.1) is a particular case of P-WLD for $\alpha=1$. It should be noted that Shanker et al. [12] have derived size-biased Poisson-weighted Lindley distribution and discussed its statistical properties, estimation of parameters using 
maximum likelihood estimation and applications. It can be easily shown that P-WLD is unimodal and has increasing hazard rate. Since

$$
\frac{P_{2}(x+1 ; \theta, \alpha)}{P_{2}(x ; \theta, \alpha)}=\left(1+\frac{\alpha-1}{x+1}\right)\left(\frac{1}{\theta+1}\right)\left[1+\frac{1}{x+\theta+\alpha+1}\right]
$$

is decreasing function in $x, \mathrm{P}_{2}(x ; \theta, \alpha)$ is log-concave. Now using the results of relationship between log-concavity, unimodality and increasing hazard rate (IHR) of discrete distributions available in literature [13], it can concluded that P-WLD has an increasing hazard rate and unimodal.

The nature and behavior of P-WLD for varying values of the parameters $\theta$ and $\alpha$ have been explained graphically in Fig. 1.
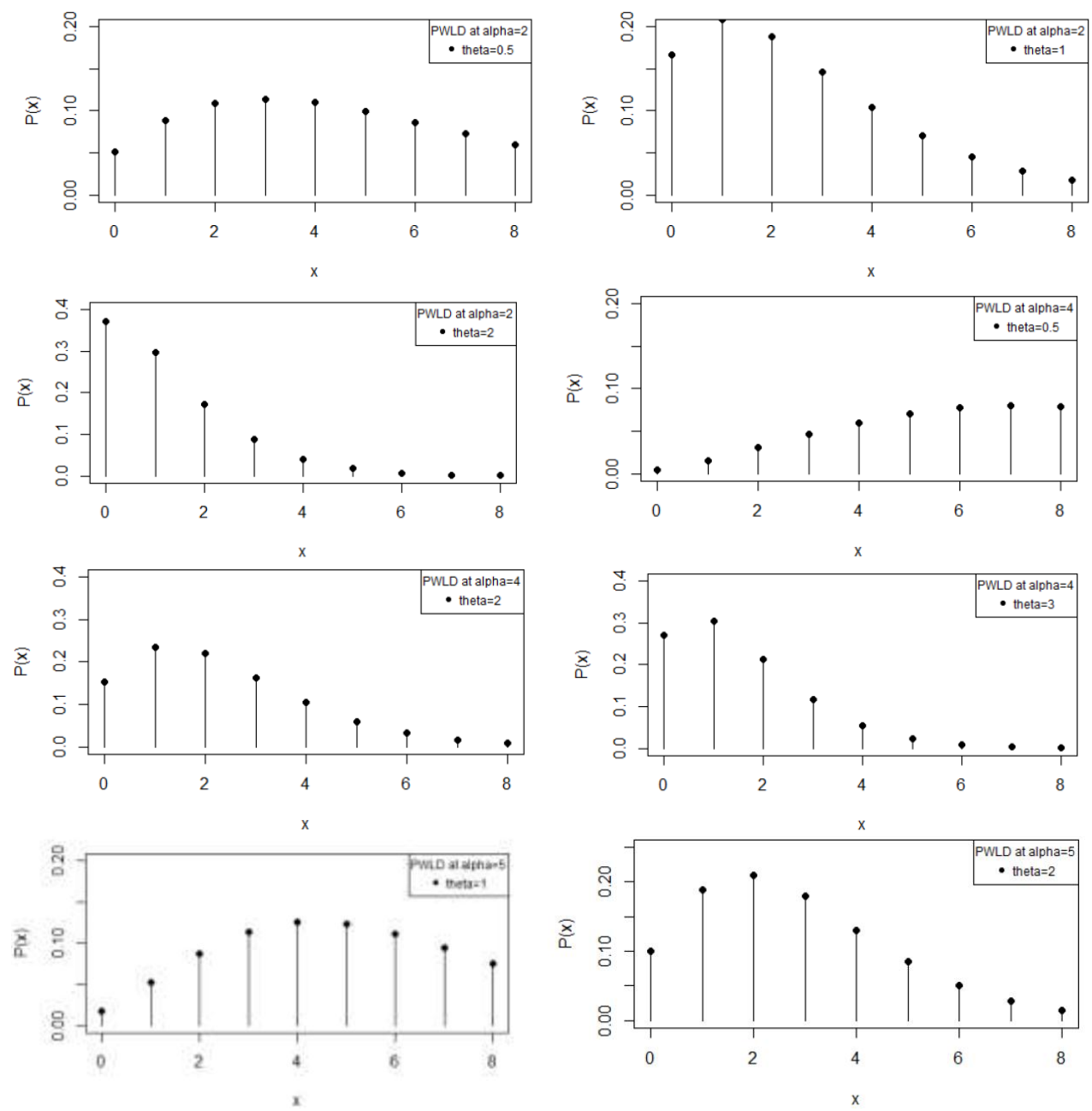

Fig. 1. Probability mass function plot of P-WLD for varying values of parameters $\theta$ and $\alpha$. 


\section{Moments, Skewness, Kurtosis and Index of Dispersion}

Using (2.1), the $r$ th factorial moment about origin of the P-WLD (2.2) can be obtained as

$$
\begin{aligned}
& \mu_{(r)}{ }^{\prime}=E\left[E\left(X^{(r)} \mid \lambda\right)\right], \text { where } X^{(r)}=X(X-1)(X-2) \ldots(X-r+1) \\
= & \int_{0}^{\infty}\left[\sum_{x=1}^{\infty} x^{(r)} \frac{e^{-\lambda} \lambda^{x}}{x !}\right] \frac{\theta^{\alpha+1}}{(\theta+\alpha)} \frac{\lambda^{\alpha-1}}{\Gamma(\alpha)}(1+\lambda) e^{-\theta \lambda} d \lambda \\
= & \frac{\theta^{\alpha+1}}{(\theta+\alpha) \Gamma(\alpha)} \int_{0}^{\infty}\left[\lambda^{r}\left\{\sum_{x=r}^{\infty} x \frac{e^{-\lambda} \lambda^{x-r}}{(x-r) !}\right\}\right] \lambda^{\alpha-1}(1+\lambda) e^{-\theta \lambda} d \lambda
\end{aligned}
$$

Taking $x-r=y$, we get

$$
\begin{aligned}
& \mu_{(r)}{ }^{\prime}=\frac{\theta^{\alpha+1}}{(\theta+\alpha) \Gamma(\alpha)} \int_{0}^{\infty}\left[\lambda^{r}\left\{\sum_{y=0}^{\infty} \frac{e^{-\lambda} \lambda^{y}}{y !}\right\}\right] \lambda^{\alpha-1}(1+\lambda) e^{-\theta \lambda} d \lambda \\
= & \frac{\theta^{\alpha+1}}{(\theta+\alpha) \Gamma(\alpha)} \int_{0}^{\infty} \lambda^{\alpha+r-1}(1+\lambda) e^{-\theta \lambda} d \lambda \\
= & \frac{(\theta+\alpha+r) \Gamma(\alpha+r)}{\theta^{r}(\theta+\alpha) \Gamma(\alpha)} ; r=1,2,3, \ldots .
\end{aligned}
$$

Taking $r=1,2,3$ and 4 in (3.1), the first four factorial moments about origin of P-WLD (2.2) can be obtained

$$
\begin{aligned}
& {\mu_{(1)}{ }^{\prime}=}=\frac{\alpha(\theta+\alpha+1)}{\theta(\theta+\alpha)} \\
& \mu_{(2)}{ }^{\prime}=\frac{\alpha(\alpha+1)(\theta+\alpha+2)}{\theta^{2}(\theta+\alpha)} \\
& \mu_{(3)}{ }^{\prime}=\frac{\alpha(\alpha+1)(\alpha+2)(\theta+\alpha+3)}{\theta^{3}(\theta+\alpha)} \\
& \mu_{(4)}{ }^{\prime}=\frac{\alpha(\alpha+1)(\alpha+2)(\alpha+3)(\theta+\alpha+4)}{\theta^{4}(\theta+\alpha)} .
\end{aligned}
$$

Now using the relationship between factorial moments about origin and the moments about origin, the first four moments about origin of P-WLD (2.2) can be obtained as

$$
\mu_{1}^{\prime}=\frac{\alpha(\theta+\alpha+1)}{\theta(\theta+\alpha)}
$$




$$
\begin{aligned}
& \mu_{2}^{\prime}= \frac{\alpha\left\{\theta^{2}+2(\alpha+1) \theta+\left(\alpha^{2}+3 \alpha+2\right)\right\}}{\theta^{2}(\theta+\alpha)} \\
& \mu_{3}^{\prime}=\frac{\alpha\left\{\theta^{3}+4(\alpha+1) \theta^{2}+4\left(\alpha^{2}+3 \alpha+2\right) \theta+\left(\alpha^{3}+6 \alpha^{2}+11 \alpha+6\right)\right\}}{\theta^{3}(\theta+\alpha)} \\
& \mu_{4}^{\prime}=\frac{\alpha\left\{\begin{array}{l}
\theta^{4}+8(\alpha+1) \theta^{3}+13\left(\alpha^{2}+3 \alpha+2\right) \theta^{2}+7\left(\alpha^{3}+6 \alpha^{2}+11 \alpha+6\right) \theta \\
+\left(\alpha^{4}+10 \alpha^{3}+35 \alpha^{2}+50 \alpha+24\right)
\end{array}\right\}}{\theta^{4}(\theta+\alpha)}
\end{aligned}
$$

Now, using the relationship $\mu_{r}=E\left(Y-\mu_{1}^{\prime}\right)^{r}=\sum_{k=0}^{r}\left(\begin{array}{l}r \\ k\end{array}\right) \mu_{k}^{\prime}\left(-\mu_{1}^{\prime}\right)^{r-k}$ between moments

about mean and the moments about origin, the moments about mean of the P-WLD (2.2) can be obtained as

$$
\begin{gathered}
\mu_{2}=\frac{\alpha\left\{\theta^{3}+2(\alpha+1) \theta^{2}+\left(\alpha^{2}+3 \alpha+2\right) \theta+\left(\alpha^{2}+\alpha\right)\right\}}{\theta^{2}(\theta+\alpha)^{2}} \\
\mu_{3}=\frac{\alpha\left\{\begin{array}{l}
\theta^{5}+(3 \alpha+4) \theta^{4}+\left(3 \alpha^{2}+11 \alpha+8\right) \theta^{3}+\left(\alpha^{3}+10 \alpha^{2}+15 \alpha+6\right) \theta^{2} \\
+3\left(\alpha^{3}+3 \alpha^{2}+2 \alpha\right) \theta+2\left(\alpha^{3}+\alpha^{2}\right)
\end{array}\right\}}{\theta^{3}(\theta+\alpha)^{3}} \\
\mu_{4}=\frac{\left\{\begin{array}{l}
\theta^{7}+(7 \alpha+8) \theta^{6}+\left(18 \alpha^{2}+43 \alpha+326\right) \theta^{5}+\left(22 \alpha^{3}+87 \alpha^{2}+107 \alpha+42\right) \theta^{4} \\
+\left(13 \alpha^{4}+83 \alpha^{3}+166 \alpha^{2}+120 \alpha+24\right) \theta^{3}+\left(3 \alpha^{5}+37 \alpha^{4}+118 \alpha^{3}+132 \alpha^{2}+48 \alpha\right) \theta^{2} \\
+\left(6 \alpha^{5}+36 \alpha^{4}+66 \alpha^{3}+36 \alpha^{2}\right) \theta+\left(3 \alpha^{5}+12 \alpha^{4}+9 \alpha^{3}\right)
\end{array}\right.}{\theta^{4}(\theta+\alpha)^{4}}
\end{gathered}
$$

The coefficient of variation $(C . V)$, coefficient of Skewness $\left(\sqrt{ } \beta_{1}\right)$, coefficient of Kurtosis $\left(\beta_{2}\right)$ and index of dispersion $(\gamma)$ of the P-WLD (2.2) are thus obtained as

$$
\begin{aligned}
& C . V=\frac{\sigma}{\mu_{1}^{\prime}}=\frac{\sqrt{\theta^{3}+2(\alpha+1) \theta^{2}+\left(\alpha^{2}+3 \alpha+2\right) \theta+\left(\alpha^{2}+\alpha\right)}}{\sqrt{\alpha}(\theta+\alpha+1)} \\
& \sqrt{\beta_{1}}=\frac{\mu_{3}}{\mu_{2}^{3 / 2}}=\frac{\left\{\begin{array}{l}
\theta^{5}+(3 \alpha+4) \theta^{4}+\left(3 \alpha^{2}+11 \alpha+8\right) \theta^{3}+\left(\alpha^{3}+10 \alpha^{2}+15 \alpha+6\right) \theta^{2} \\
+3\left(\alpha^{3}+3 \alpha^{2}+2 \alpha\right) \theta+2\left(\alpha^{3}+\alpha^{2}\right)
\end{array}\right.}{\sqrt{\alpha}\left\{\theta^{3}+2(\alpha+1) \theta^{2}+\left(\alpha^{2}+3 \alpha+2\right) \theta+\left(\alpha^{2}+\alpha\right)\right\}^{3 / 2}}
\end{aligned}
$$




$$
\begin{aligned}
& \beta_{2}=\frac{\mu_{4}}{\mu_{2}^{2}}=\frac{\left\{\begin{array}{l}
\theta^{7}+(7 \alpha+8) \theta^{6}+\left(18 \alpha^{2}+43 \alpha+326\right) \theta^{5}+\left(22 \alpha^{3}+87 \alpha^{2}+107 \alpha+42\right) \theta^{4} \\
+\left(13 \alpha^{4}+83 \alpha^{3}+166 \alpha^{2}+120 \alpha+24\right) \theta^{3}+\left(3 \alpha^{5}+37 \alpha^{4}+118 \alpha^{3}+132 \alpha^{2}+48 \alpha\right) \theta^{2} \\
+\left(6 \alpha^{5}+36 \alpha^{4}+66 \alpha^{3}+36 \alpha^{2}\right) \theta+\left(3 \alpha^{5}+12 \alpha^{4}+9 \alpha^{3}\right)
\end{array}\right\}}{\alpha\left\{\theta^{3}+2(\alpha+1) \theta^{2}+\left(\alpha^{2}+3 \alpha+2\right) \theta+\left(\alpha^{2}+\alpha\right)\right\}^{2}} \\
& \gamma=\frac{\sigma^{2}}{\mu_{1}^{\prime}}=\frac{\left\{\theta^{3}+2(\alpha+1) \theta^{2}+\left(\alpha^{2}+3 \alpha+2\right) \theta+\left(\alpha^{2}+\alpha\right)\right\}}{\theta(\theta+\alpha)(\theta+\alpha+1)} .
\end{aligned}
$$

Nature and behavior of coefficient of variation, coefficient of skewness, coefficient of kurtosis and index of dispersion of P-WLD for varying values of parameters $\theta$ and $\alpha$ have been shown graphically in Fig. 2.
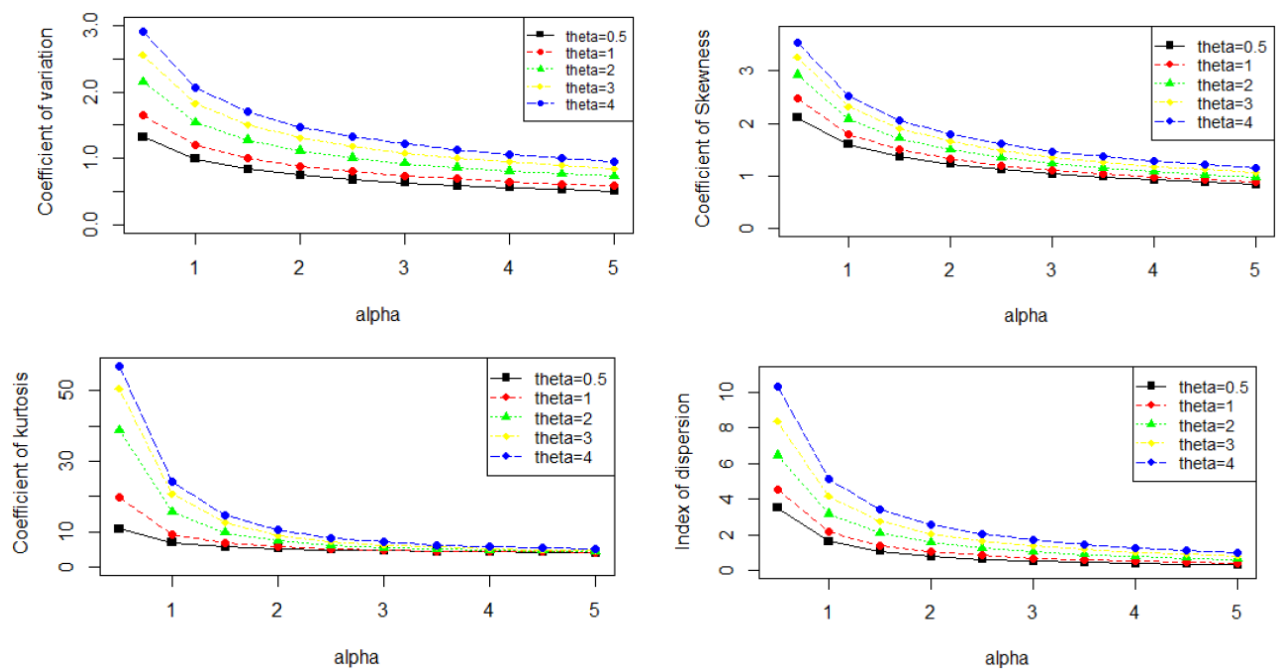

Fig. 2. Nature and behavior of coefficient of variation, coefficient of skewness, coefficient of kurtosis and index of dispersion of P-WLD for varying values of parameters $\theta$ and $\alpha$.

\section{Maximum Likelihood Estimation}

Let $\left(x_{1}, x_{2}, \ldots \ldots x_{\mathrm{n}}\right)$ be a random sample of size $n$ from the P-WLD (2.2) and let $f_{x}$ be the observed frequency in the sample corresponding to $X=x(x=1,2,3, \ldots k)$ such that $\sum_{x=1}^{k} f_{x}=n$, where $k$ is the largest observed value having non-zero frequency. The log likelihood function of P-WLD (2.2) can be given by 


$$
\begin{aligned}
\log L= & n[(\alpha+1) \log \theta-\log (\theta+\alpha)]-\sum_{x=1}^{k} f_{x}(x+\alpha+1) \log (\theta+1)+\sum_{x=1}^{k} f_{x} \log (x+\theta+\alpha+1) \\
& +\sum_{x=1}^{k} f_{x}[\log \Gamma(x+\alpha)-\log \Gamma(\alpha)-\log (x+1)]
\end{aligned}
$$

The maximum likelihood estimates $(\hat{\theta}, \hat{\alpha})$ of $(\theta \alpha)$ of P-WLD (2.2) is the solutions of the following log likelihood equations

$$
\frac{\partial \log L}{\partial \theta}=\frac{n(\alpha+1)}{\theta}-\frac{n}{\theta+\alpha}-\sum_{x=1}^{k} \frac{(x+\alpha+1) f_{x}}{\theta+1}+\sum_{x=1}^{k} \frac{f_{x}}{x+\theta+\alpha+1}=0
$$

$\frac{\partial \log L}{\partial \alpha}=n \log \theta-\frac{n}{\theta+\alpha}-\sum_{x=1}^{k} f_{x} \log (\theta+1)+\sum_{x=1}^{k} \frac{f_{x}}{x+\theta+\alpha+1}+\sum_{x=1}^{k} f_{x}[\psi(x+\alpha)-\psi(\alpha)]=0$ where $\bar{x}$ is the sample mean and $\psi(x+\alpha)=\frac{d}{d \alpha} \log \Gamma(x+\alpha)$ and $\psi(\alpha)=\frac{d}{d \alpha} \log \Gamma(\alpha)$ are digamma functions.

These two log likelihood equations do not seem to be solved directly. However, the Fisher's scoring method can be applied to solve these equations. We have

$$
\begin{aligned}
& \frac{\partial^{2} \log L}{\partial \theta^{2}}=-\frac{n(\alpha+1)}{\theta^{2}}+\frac{n}{(\theta+\alpha)^{2}}+\sum_{x=1}^{k} \frac{(x+\alpha+1) f_{x}}{(\theta+1)^{2}}-\sum_{x=1}^{k} \frac{f_{x}}{(x+\theta+\alpha+1)^{2}} \\
& \frac{\partial^{2} \log L}{\partial \alpha^{2}}=\frac{n}{(\theta+\alpha)^{2}}-\sum_{x=1}^{k} \frac{f_{x}}{(x+\theta+\alpha+1)^{2}}+\sum_{x=1}^{k} f_{x}\left[\psi^{\prime}(x+\alpha)-\psi^{\prime}(\alpha)\right] \\
& \frac{\partial^{2} \log L}{\partial \theta \partial \alpha}=\frac{n}{\theta}+\frac{n}{(\theta+\alpha)^{2}}-\sum_{x=1}^{k} \frac{f_{x}}{\theta+1}-\sum_{x=1}^{k} \frac{f_{x}}{(x+\theta+\alpha+1)^{2}}=\frac{\partial^{2} \log L}{\partial \alpha \partial \theta},
\end{aligned}
$$

Where $\psi^{\prime}(x+\alpha)=\frac{d}{d \alpha} \psi(x+\alpha)$ and $\psi^{\prime}(\alpha+1)=\frac{d}{d \alpha} \psi(\alpha+1)$ are trigamma functions.

The maximum likelihood estimates $(\hat{\theta}, \hat{\alpha})$ of $(\theta \alpha)$ of P-WLD (2.2) is the solution of the following equations

$\left[\begin{array}{ll}\frac{\partial^{2} \log L}{\partial \theta^{2}} & \frac{\partial^{2} \log L}{\partial \theta \partial \alpha} \\ \frac{\partial^{2} \log L}{\partial \alpha \partial \theta} & \frac{\partial^{2} \log L}{\partial \alpha^{2}}\end{array}\right]_{\substack{\hat{\theta}=\theta_{0} \\ \hat{\alpha}=\alpha_{0}}}\left[\begin{array}{c}\hat{\theta}-\theta_{0} \\ \hat{\alpha}-\alpha_{0}\end{array}\right]=\left[\begin{array}{l}\frac{\partial \log L}{\partial \theta} \\ \frac{\partial \log L}{\partial \alpha}\end{array}\right]_{\substack{\hat{\theta}=\theta_{0} \\ \hat{\alpha}=\alpha_{0}}}$

Where $\theta_{o}$ and $\alpha_{o}$ are the initial values of $\theta$ and $\alpha$ respectively. These equations are solved iteratively till sufficiently close values of $\widehat{\theta}$ and $\widehat{\alpha}$ are obtained. 


\section{Applications}

It would be worth to mention that there are several mistakes regarding applications in the paper [11], namely (i) the major problem of their paper is that although they claim that $\mathrm{P}$ WLD fits well but the fit of P-WLD is not better than one parameter PLD and Twoparameter GPLD, (ii) the ML estimates of the parameter of Hermite distribution is not given, (iii) the pmf of GPLD have parameters $\theta$ and $\alpha$ but in the goodness of fit they mentioned $c$ and $\theta$, (iv) the application and the conclusion of the paper are not correct. In fact the paper [11] is full of mistakes and typing errors.

In this section the applications of the P-WLD has been discussed with some count datasets from biological sciences and thunderstorms events. The dataset in Table 1 is the data regarding the number of European red motes on apple leaves, available in reference [14]. The dataset in Tables 2 and 3 are the Mammalian Cytogenetic dosimetry Lesions in Rabbit Lymphoblast induced by streponigrin (NSC-45383), available in reference [15]. The dataset in Table 4 is the number of micronuclei after exposure at dose 4 Gy of jirradiation, counted using the cytochalasin B method and available in reference [16]. The dataset in Tables 5 and 6 are the frequencies of the observed number of days that experienced $\mathrm{X}$ thunderstorm events at Cape kennedy, Florida for the 11-year period of record in the month of June and July, January 1957 to December 1967 and are available in references [17,18]. The goodness of fit of P-WLD has been compared with the goodness of fit given by Poisson distribution (PD), PLD, negative binomial distribution (NBD) and GPLD. Note that the estimates of the parameters are based on maximum likelihood estimates for all the considered distributions. Based on the values of chi-square $\left({ }^{2}\right),-2 \log \mathrm{L}$ and AIC (Akaike Information criterion), it is obvious that P-WLD is competing well with the considered distributions. Note that AIC has been calculated using the formula $A I C=-2 \log L+2 k$, where $k$, is the number of parameters involved in the distribution.

In Table 1, P-WLD gives better fit than PD, PLD, NBD and GPLD. In Table 2, PWLD gives better fit than PD, PLD, NBD and GPLD. In Table 3, PLD Gives better fit than PD, NBD, GPLD and P-WLD. In Table 4, NBD and GPLD are almost gives the same fit but better than PD, PLD and P-WLD. In Table 5, PLD, GPLD, and P-WLD are almost the same but better than PD and NBD. In Table 6, PLD gives better fit than PD, NBD, GPLD and P-WLD. Therefore, it can be concluded that P-WLD is competing well with PD, PLD, NBD, and GPLD, and thus it can be considered an important distribution. 
Table 1. Observed and Expected number of European red mites on Apple leaves, available in reference [15].

\begin{tabular}{lllllll}
\hline \multirow{2}{*}{$\begin{array}{c}\text { Number of } \\
\text { European red } \\
\text { mites per leaf }\end{array}$} & $\begin{array}{c}\text { Observed } \\
\text { frequency }\end{array}$ & \multicolumn{5}{c}{ Expected frequency } \\
\cline { 2 - 7 } & 70 & PD & PLD & NBD & GPLD & P-WLD \\
\hline 0 & 38 & 54.6 & 38.9 & 37.6 & 36.8 & 69.8 \\
1 & 17 & 31.3 & 21.2 & 20.1 & 20.1 & 20.1 \\
2 & 10 & 11.9 & 11.1 & 10.7 & 10.9 & 10.9 \\
3 & 9 & 3.4 & 5.7 & 5.7 & 5.8 & 5.8 \\
4 & 3 & 0.8 & 2.8 & 3.0 & 3.1 & 3.0 \\
5 & 2 & 0.2 & 1.4 & 1.6 & 1.6 & 1.6 \\
6 & 1 & 0.1 & 0.9 & 0.9 & 0.8 & 0.8 \\
7 & 0.1 & 0.8 & 0.9 & 1.2 & 1.2 \\
8 & 150.0 & 150.0 & 150.0 & 150.0 & 150.0 \\
Total & 150 & & $\hat{\theta}=1.26010$ & $\hat{\alpha}=1.02459$ & $\hat{\theta}=1.09620$ & $\hat{\theta}=1.09141$ \\
ML & $\hat{\theta}=1.14666$ & & & $\widehat{P}=0.52811$ & $\hat{\alpha}=0.78005$ & $\hat{\alpha}=0.82194$ \\
estimate & & & & & & \\
Standard $\hat{\theta}$ & & 0.08743 & 0.11390 & 0.42097 & 0.25400 & 0.26231 \\
Errors $\hat{\alpha}$ & & & & 0.40136 & 0.31550 & 0.25230 \\
$\left({ }^{2}\right)$ & & 26.50 & 2.49 & 2.91 & 2.43 & 2.41 \\
d.f & & 2 & 4 & 3 & 3 & 3 \\
p-value & & 0.0000 & 0.5595 & 0.4057 & 0.4880 & 0.4917 \\
-2logL & & 485.61 & 445.02 & 469.68 & 444.62 & 425.35 \\
AIC & & 487.61 & 447.02 & 447.02 & 448.62 & 429.35 \\
\hline
\end{tabular}

Table 2. Mammalian Cytogenetic dosimetry Lesions in Rabbit Lymphoblast induced by streponigrin (NSC-45383), exposure- $60 \mu \mathrm{g} / \mathrm{kg}$.

\begin{tabular}{lllllll}
\hline \multirow{2}{*}{$\begin{array}{l}\text { Class/Exposure } \\
(\mu \mathrm{g} / \mathrm{kg})\end{array}$} & $\begin{array}{l}\text { Observed } \\
\text { frequency }\end{array}$ & \multicolumn{5}{c}{ Expected frequency } \\
\cline { 5 - 7 } & & $\mathrm{PD}$ & $\mathrm{PLD}$ & $\mathrm{NBD}$ & $\mathrm{GPLD}$ & $\mathrm{P}-\mathrm{WLD}$ \\
\hline 0 & 413 & 374.0 & 405.7 & 412.7 & 412.9 & 412.9 \\
1 & 124 & 177.4 & 133.6 & 124.9 & 124.1 & 124.3 \\
2 & 42 & 42.1 & 42.6 & 41.5 & 42.0 & 41.9 \\
3 & 15 & 6.6 & 13.3 & 14.2 & 14.3 & 14.4 \\
4 & 5 & 0.8 & 4.1 & 4.9 & 4.97 & 4.9 \\
5 & 0 & 0.1 & 1.2 & 1.7 & 1.6 & 1.7 \\
6 & 2 & 0.0 & 0.5 & 1.1 & 1.2 & 0.9 \\
Total & 601 & 601.0 & 601.0 & 601.0 & 601.0 & 601.0 \\
ML & & $\hat{\theta}=0.47421$ & $\hat{\theta}=2.68537$ & $\hat{\theta}=1.76494$ & $\hat{\theta}=2.16876$ & $\hat{\theta}=2.12567$ \\
estimate & & & & $\hat{\alpha}=0.83700$ & $\hat{\alpha}=0.71287$ & $\hat{\alpha}=0.74791$ \\
Standard $\hat{\theta}$ & & 0.02809 & 0.16467 & 0.40075 & 0.38481 & 0.41314 \\
Errors $\hat{\alpha}$ & & & & 0.17964 & 0.20487 & 0.17437 \\
$\left({ }^{2}\right)$ & & 48.169 & 1.336 & 0.12 & 0.098 & 0.059 \\
d.f & & 2 & 3 & 2 & 2 & 2 \\
p-value & & 0.0000 & 0.7206 & 0.94129 & 0.9520 & 0.9709 \\
-2logL & & 1165.35 & 1113.76 & 1112.39 & 1112.36 & 1271.94 \\
AIC & & 1167.35 & 1115.76 & 1116.39 & 1116.36 & 1275.94 \\
\hline
\end{tabular}


Table 3. Mammalian Cytogenetic dosimetry Lesions in Rabbit Lymphoblast induced by streponigrin (NSC-45383), exposure- $90 \mu \mathrm{g} / \mathrm{kg}$.

\begin{tabular}{|c|c|c|c|c|c|c|}
\hline \multirow{2}{*}{$\begin{array}{l}\text { Class/Exposure } \\
(\mu \mathrm{g} / \mathrm{kg})\end{array}$} & \multirow{2}{*}{$\begin{array}{l}\text { Observed } \\
\text { frequency }\end{array}$} & \multicolumn{5}{|c|}{ Expected frequency } \\
\hline & & $\mathrm{PD}$ & PLD & NBD & GPLD & P-WLD \\
\hline 0 & 155 & 127.8 & 158.3 & 155.1 & 155.3 & 155.9 \\
\hline 1 & 83 & 109.0 & 77.2 & 80.6 & 80.1 & 80.0 \\
\hline 2 & 33 & 46.5 & 35.9 & 36.7 & 36.9 & 36.7 \\
\hline 3 & 14 & 13.2 & 16.1 & 15.9 & 16.0 & 15.9 \\
\hline 4 & 11 & 2.8 & 7.1 & 6.7 & 6.7 & 6.7 \\
\hline 5 & 3 & 0.5 & 3.1 & 2.8 & 2.8 & 2.7 \\
\hline 6 & 1 & 0.2 & 2.3 & 2.2 & 2.2 & 2.1 \\
\hline Total & 300 & 300.0 & 300.0 & 300.0 & 300.0 & 300.0 \\
\hline ML & & $\widehat{\theta}=0.85333$ & $\widehat{\theta}=1.61761$ & $\widehat{\theta}=1.56009$ & $\hat{\theta}=1.80860$ & $\widehat{\theta}=1.82011$ \\
\hline estimate & & & & $\hat{\alpha}=1.33128$ & $\hat{\alpha}=1.18743$ & $\hat{\alpha}=1.16320$ \\
\hline Standard $\hat{\theta}$ & & 0.05333 & 0.11327 & 0.41479 & 0.40045 & 0.41992 \\
\hline Errors $\hat{\alpha}$ & & & & 0.33752 & 0.37007 & 0.32483 \\
\hline$\left({ }^{2}\right)$ & & 24.969 & 1.51 & 1.60 & 1.69 & 1.78 \\
\hline d.f & & 2 & 3 & 2 & 2 & 2 \\
\hline$p$-value & & 0.0000 & 0.6799 & 0.4488 & 0.42955 & 0.4106 \\
\hline$-2 \log L$ & & 800.92 & 766.10 & 765.86 & 765.79 & 834.51 \\
\hline AIC & & 802.92 & 768.10 & 769.86 & 769.79 & 838.51 \\
\hline
\end{tabular}

Table 4. Number of micronuclei after exposure at dose 4 Gy of $\gamma$ irradiation, counted using the cytochalasin B method and available in reference [16].

\begin{tabular}{|c|c|c|c|c|c|c|}
\hline \multirow{2}{*}{$\begin{array}{l}\text { Number of } \\
\text { micronuclei }\end{array}$} & \multirow{2}{*}{$\begin{array}{l}\text { Observed } \\
\text { frequency }\end{array}$} & \multicolumn{5}{|c|}{ Expected frequency } \\
\hline & & PD & PLD & NBD & GPLD & P-WLD \\
\hline 0 & 1974 & 1816.0 & 2396.8 & 1966.2 & 1964.9 & 1966.0 \\
\hline 1 & 1674 & 1839.9 & 1300.3 & 1695.5 & 1696.6 & 1695.5 \\
\hline 2 & 869 & 932.1 & 668.8 & 331.5 & 857.9 & 857.6 \\
\hline 3 & 342 & 314.8 & 332.1 & 857.5 & 331.5 & 331.7 \\
\hline 4 & 102 & 79.7 & 160.9 & 108.4 & 108.3 & 108.5 \\
\hline 5 & 26 & 16.1 & 76.5 & 31.6 & 31.5 & 31.5 \\
\hline 6 & 13 & 2.7 & 35.8 & 8.4 & 8.41 & 8.4 \\
\hline 7 & 2 & 1.6 & 30.8 & 2.9 & 2.9 & 2.8 \\
\hline Total & 5002 & 5002.0 & 5002.0 & 5002.0 & 5002.0 & 5002.0 \\
\hline ML & & $\hat{\theta}=1.01319$ & $\hat{\theta}=1.38736$ & 5.71671 & $\hat{\theta}=5.88560$ & $\hat{\theta}=5.97458$ \\
\hline estimate & & & & 5.79197 & $\hat{\alpha}=5.81844$ & $\hat{\alpha}=5.57089$ \\
\hline Standard $\hat{\theta}$ & & 0.01423 & 0.02251 & 0.82644 & 0.82310 & 0.83470 \\
\hline Errors $\hat{\alpha}$ & & & & 0.83264 & 0.84660 & 0.83790 \\
\hline$\left({ }^{2}\right)$ & & 62.21 & 337.08 & 3.37 & 3.36 & 3.41 \\
\hline d.f & & 4 & 5 & 4 & 4 & 4 \\
\hline p-value & & 0.0000 & 0.0000 & 0.4976 & 0.4995 & 0.4909 \\
\hline$-2 \log L$ & & 13535.82 & 13836.70 & 13471.80 & 13471.81 & 15597.78 \\
\hline AIC & & 13537.82 & 13838.70 & 13475.80 & 13475.81 & 15601.78 \\
\hline
\end{tabular}


Table 5. Frequencies of the observed number of days that experienced $X$ thunderstorm events at Cape kennedy, Florida for the 11-year period of record in the month of June, January 1957 to December 1967.

\begin{tabular}{lllllll}
\hline \multirow{2}{*}{$\mathrm{X}$} & \multirow{2}{*}{$\begin{array}{l}\text { Observed } \\
\text { frequency }\end{array}$} & \multicolumn{5}{c}{ Expected frequency } \\
\cline { 3 - 7 } & 187 & 155.6 & 185.3 & 184.6 & 185.3 & 185.1 \\
\hline 0 & 77 & 116.9 & 83.4 & 84.5 & 83.5 & 83.7 \\
1 & 40 & 43.9 & 35.9 & 35.8 & 35.9 & 36.0 \\
2 & 17 & 11.0 & 15.0 & 14.8 & 15.0 & 15.0 \\
3 & 6 & 2.0 & 6.1 & 6.0 & 6.1 & 6.1 \\
4 & 2 & 0.3 & 2.5 & 2.4 & 2.5 & 2.4 \\
5 & 1 & 0.3 & 1.8 & 1.9 & 1.7 & 1.7 \\
6 & 330 & 330.0 & 330.0 & 330.0 & 330.0 & 330.0 \\
Total & & $\hat{\theta}=0.75148$ & $\hat{\theta}=1.80427$ & $\hat{\theta}=1.55916$ & $\hat{\theta}=1.80780$ & $\hat{\theta}=1.82188$ \\
ML & & & & $\hat{\alpha}=1.17172$ & $\hat{\alpha}=1.00340$ & $\hat{\alpha}=1.01237$ \\
estimate & & 0.04772 & 0.12573 & 0.41501 & 0.39558 & 0.41748 \\
Standard $\hat{\theta}$ & & & & 0.29696 & 0.32657 & 0.28219 \\
Errors $\hat{\alpha}$ & & 31.6 & 1.43 & 1.68 & 1.42 & 1.41 \\
$\left({ }^{2}\right)$ & & 2 & 3 & 2 & 2 & 2 \\
d.f & & 0.0000 & 0.6985 & 0.4317 & 0.4916 & 0.4941 \\
p-value & & 824.50 & 788.88 & 789.18 & 788.88 & 874.20 \\
-2logL & & 826.50 & 790.88 & 793.18 & 792.88 & 878.20 \\
AIC & & & & & & \\
\hline
\end{tabular}

Table 6. Frequencies of the observed number of days that experienced $X$ thunderstorm events at Cape kennedy, Florida for the 11-year period of record in the month of July, January 1957 to December 1967.

\begin{tabular}{|c|c|c|c|c|c|c|}
\hline \multirow{2}{*}{$X$} & \multirow{2}{*}{$\begin{array}{l}\text { Observed } \\
\text { frequency }\end{array}$} & \multicolumn{5}{|c|}{ Expected frequency } \\
\hline & & PD & PLD & NBD & GPLD & P-WLD \\
\hline 0 & 177 & 142.3 & 177.7 & 171.8 & 172.7 & 172.5 \\
\hline 1 & 80 & 124.3 & 87.9 & 94.0 & 92.8 & 92.9 \\
\hline 2 & 47 & 54.3 & 41.5 & 43.3 & 43.2 & 43.3 \\
\hline 3 & 26 & 15.8 & 18.9 & 18.7 & 18.8 & 18.9 \\
\hline 4 & 9 & 3.5 & 8.4 & 7.8 & 8.0 & 7.9 \\
\hline 5 & 2 & 0.8 & 6.6 & 5.4 & 5.4 & 5.5 \\
\hline Total & 341 & 341.0 & 341.0 & 341.0 & 341.0 & 341.0 \\
\hline ML & & $\hat{\theta}=0.87390$ & $\hat{\theta}=1.58353$ & $\hat{\theta}=1.67672$ & $\hat{\theta}=1.86350$ & $\widehat{\theta}=1.89198$ \\
\hline estimate & & & & $\hat{\alpha}=1.46527$ & $\hat{\alpha}=1.28028$ & $\hat{\alpha}=1.25544$ \\
\hline Standard $\hat{\theta}$ & & 0.05062 & 0.10317 & 0.45068 & 0.42561 & 0.44313 \\
\hline Errors $\hat{\alpha}$ & & & & 0.37896 & 0.40429 & 0.35588 \\
\hline$\left({ }^{2}\right)$ & & 39.40 & 5.16 & 5.77 & 5.39 & 5.32 \\
\hline d.f & & 2 & 3 & 2 & 2 & 2 \\
\hline p-value & & 0.0000 & 0.1594 & 0.0558 & 0.0674 & 0.0699 \\
\hline$-2 \log \mathrm{L}$ & & 911.00 & 880.50 & 880.35 & 879.93 & 967.56 \\
\hline $\mathrm{AIC}$ & & 913.00 & 882.50 & 884.35 & 883.93 & 971.56 \\
\hline
\end{tabular}




\section{Conclusion}

In this paper a Poisson-weighted Lindley distribution (P-WLD), a Poisson mixture of weighted Lindley distribution has been proposed and its nature and behavior have been discussed graphically. The nature and behavior of its coefficient of variation, skewness, kurtosis and index of dispersion have been explained graphically. Maximum likelihood estimation has been discussed to estimate its parameters. Applications of the proposed distribution have been discussed and its goodness of fit has been compared with Poisson distribution (PD), Poisson-Lindley distribution (PLD), negative binomial distribution (NBD) and generalized Poisson-Lindley distribution (GPLD) and it has been observed that P-WLD is competing well with the considered distributions.

\section{Acknowledgments}

Authors are grateful to the editor in chief of the journal and anonymous reviewer for fruitful comments on the paper which improved the quality of the paper.

\section{References}

1. M. Sankaran, Biometrics 26, 145 (1970). https://doi.org/10.2307/2529053

2. R. Shanker, and F. Hagos, Biomet. Biostat. Int. J. 2, 1 (2015).

3. R. Shanker and K. K. Shukla, J. Sci. Res. 10, 145 (2018). https://doi.org/10.3329/jsr.v10i2.34905

4. M. E. Ghitany and D. K. Al-Mutairi, MetronInt. J. Stat. 66, 299 (2008).

5. D. Vlindley, J. Royal Stat. Soc. B 20, 102 (1958).

6. M. E. Ghitany, B. Atieh, and S. Nadarajah, Math. Comput. Simul.78, 493 (2008). https://doi.org/10.1016/j.matcom.2007.06.007

7. R. Shanker, F. Hagos, and S. Sujatha, Biomet. Biostat. Int. J. 2, 1 (2015).

8. M. E. Ghitany, F. Alqallaf, D. K. Al-Mutairi, and H. A. Husain, Math. Comput. Simul. 81, 1190 (2011). https://doi.org/10.1016/j.matcom.2010.11.005

9. R. Shanker, K. K. Shukla, and F. Hagos, Jacobs J. Biostat. 1, 1 (2016).

10. R. Shanker, K. K.Shukla, and A. Mishra, Statistics Transition-New Series 18, 291 (2017). https://doi.org/10.21307/stattrans-2016-071

11. M. M. E. El-Monsef and N. M. Sohsah, Jokull J. 64, 192 (2014).

12. R. Shanker and K. K. Shukla, Int. J. Stat. Appl. Math. 3, 146 (2018).

13. J. Grandell, Mixed Poisson Processes (Chapman \& Hall, London, 1997). https://doi.org/10.1007/978-1-4899-3117-7

14. C. I. Bliss, Biometrics 9, 177 (1953). https://doi.org/10.2307/3001850

15. D. G. Catcheside, D. E. Lea, and J. M. Thoday, J. Genetics 47, 137 (1946). https://doi.org/10.1007/BF02986782

16. P. Puig and J. Valero, J. Am. Stat. Assoc. 101, 332 (2006). https://doi.org/10.1198/016214505000000718

17. L. W. Falls, W. O. Wilford, and M. C. Carter, J. Appl. Meteorology 10, 97 (1971). https://doi.org/10.1175/1520-0450(1971)010<0097:PDFTAA>2.0.CO;2

18. M. C. Carter, J. Royal Stat. Soc. C (Applied Statistics) 2, 196 (2001). 